\title{
Availability Assessment of Electric Power based on Switch Reliability Modelling with Dynamic Bayesian Networks: Case Study of Electrical Distribution Networks
}

\author{
Abdelaziz Lakehal $^{1}$, Zine Ghemari ${ }^{2}$ \\ 1. Department of Mechanical Engineering Mohamed Chérif Messaadia University, P.O. Box 1553, Souk-Ahras 41000, Algeria. \\ 2. Electrical Engineering Department, University of M'sila, M'sila, Algeria.
}

Received: March 26, 2015 / Accepted: April 24, 2015 / Published: July 25, 2015.

\begin{abstract}
As a generalization of the successful hidden Markov models, Dynamic Bayesian Networks (DBNs) are a natural basis for the general temporal action interpretation task. This document provides a conditional probabilistic approach to analyze the energy availability in electrical distribution networks by using Bayesian networks (BN). Firstly a static BN modelling is presented to show the influence of the switch behaviour on the energy availability. Then, the dynamic behaviour of the switch is cared by switch reliability modelling using DBN which permits to predict the energy availability. The prediction by DBNs discussed in the case study of this paper gives a strong contribution on electrical network supervisory control and it can also be applied to transportation networks.
\end{abstract}

\section{Introduction}

An electrical distribution network depends on an operator that must ensure the operation and maintenance while ensuring the quality and continuity of supply, security of persons and property. Operating the network will lead to react to the result of various events occurring on this network. The fact that events are random, it is necessary to determine how to behave according to the situations, imagine the maximum failure scenarios to predict failures and ensure optimal network availability. The Bayesian networks (BNs) proposed in this paper were used in many fields and to resolve different problems. Modelling with static BN is similar to that of fault tree [1]. [2] have used BNs for estimation of overhead

Corresponding author: Abdelaziz Lakehal, Ph.D., research fields: maintenance, Bayesian networks, power system. E-mail: a.lakehal@univ-soukahras.dz, lakehal21@yahoo.fr. lines failure rate in electrical distribution systems. [3] showed that employing Bayesian methods could enhance transmission grid risk analysis. [4] used Bayesian method for electrical power system transient stability assessment. [5] developed a Bayesian belief network model for electrical load demand.

Geographic knowledge of network, topology and the electrical state knowledge of the network are necessary to master situations that occur each time one or more sections of the network are unavailable [6]. The operations manager must then seek to restore power supply of the largest possible number of customers taking into account any statutory priorities or the sensivity of customers regarding power outage. To do this in the best conditions, it is essential to prepare troubleshooting plans that allow designing in advance of the maneuver sequences to restore power. 
Among all constituent elements of an electrical distribution network, the switch is the most important element of maneuver. It has a dynamic behaviour. It ensures isolation and power restoration function. It performs the function of isolation and power restoration; in this case the maintenance over time of these two functions is essential. The reliability of a switch is the maintaining over the time of the isolation and restoration performances.

This paper presents an approach for assessing the availability of electric power based on reliability modelling of switching devices using DBNs. The remainder of this paper is organized as follows: In section 2, BNs are presented. Section 3 presents the research methodology. Section 4 is devoted to the application of our approach to an electrical distribution network supplying a city. Finally, conclusions are presented at section 5 .

\section{Bayesian Networks}

Probabilistic graphical models such as BNs [7] have been widely used to solve various problems (for example, diagnosis, classification, failure prediction and risk analysis) [8, 11]. These models are characterized by their ability to treat uncertain information and represent the interdependencies between different variables of a given problem. The advantage of probabilistic graphical models is interesting graphical representation of models, easy to understand and analyze. In addition, the probabilistic failure analysis evaluates the probability of failure of a complex system that its weak points can be identified.

In Bayesian Network (BN), indicating an arc between two variables implies direct dependence between these two variables (one is the parent and the other is the child). It must provide the behavior of the child variable regarding the behavior of its Parents. For this, each node has a conditional probability table (CPT). A CPT associated with a node enabling to quantify the effect of parents' nodes on this node: it describes the probabilities associated with child nodes according to the different values of the parents' nodes. For root nodes (without parents), the probability table is no more conditional and then, fixes a priori probabilities concerning the variable values [7].

BNs prohibit child dependencies of parents. Thus, the set of variables and arcs will form a directed graph (arcs have a direction), and acyclic (no cycles in the graph). So a BN (Fig. 1) is defined by a directed acyclic graph (DAG), as explained in [12]:

$$
p\left(V_{1}, V_{2}, \ldots ., V_{n}\right)=\prod_{i=1}^{n} p\left(V_{i} / C\left(V_{i}\right)\right)
$$

Where $\mathrm{C}\left(\mathrm{V}_{\mathrm{i}}\right)$ is the set of parents (or causes) of $\mathrm{V}_{\mathrm{i}}$ in DAG.

\section{Methodology}

A $\mathrm{BN}$ is a modeling tool which deals issues where the variable is static. In such a field each variable has a unique and fixed value. Unfortunately this hypothesis of static world does not always take, many fields exist where the variables are dynamic and reasoning over time is necessary, such as dynamic systems. DBN are graphical models for representing compactly the uncertainties inherent in dynamic systems evolving with time. The BNs and DBNs provided a strong contribution in the field of reliability. For example the work of [13] is cited for reliability modelling of a complex system using BNs, and the work of [14] for modelling reliability with DBNs.

In this study we will use DBNs to assess the reliability of switch, and consequently predict the different situations of power supply. The study approach was inspired primarily from the work of [14]. In order to control the electrical distribution network, we must control and monitor the evolution over the time of the variables (control of section) for each section. To achieve this objective the idea is to infer,

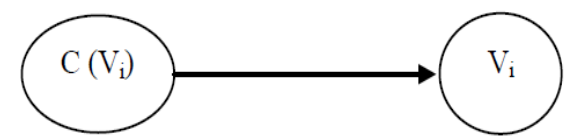

Fig. 3 Graphical representation of a simple BN 
what is the possibility for example that a section is isolated or supplied based on the switch closing and opening sequences. In this case the hidden random variable is (control of section) $)_{t}$ with two state (isolated section) $)_{\mathrm{t}}$ and (section supplied) $)_{\mathrm{t}}$, and the observed variable is (state of switch)t. the satisfaction of these suppositions is modelled using the dependencies between all model variables that which is given by the DBN of Fig. 2 :

In Fig. 2, the fact indicate an arc between the two variables control of section) $)_{\mathrm{t}}$ and (state of switch), this means that the isolation and power restoration of section depends on the switch condition and its reliability at time $t$, and even the switch reliability at time $t$ depends on its reliability at time $t-1$.

From this DBN it is possible to calculate the most recent a posteriori distribution of the variable (control section) $)_{\mathrm{t}}$ with filtering, as it is possible to calculate the a posteriori probability in a future time of variable (control section $)_{t+i}$, where $i$ is the number of time steps as given by formula (2):

$$
P\left(V_{t} / V_{t-1}\right)=\prod_{i=1}^{N} P\left(V_{i, t} / C\left(V_{i}\right)_{i, t}\right)
$$

\section{Case Study}

The electrical network supplied a locality is used as a case study network to show the strong contribution of BNs in electrical distribution networks operations. This network is supplied at 30KVA (Fig. 3).

The protection of this network is provided by a switch remote-controlled to voltage dips (interrupteur aérien télécommandé à creux de tension (IATCT)), installed in tap-off head and eliminates it in case of default, opening automatically during the slow cycle breaker tripping out. These switches allow for easy location of permanent faults and reduce sections unpowered during repairs to the following incidents or when scheduled, if they cannot be carried out under tension.

\subsection{Static BN modelling}

In this study we are interested in the reliability of the switches that allow the isolation and the current restoration of the various network sections. To control an electrical distribution network must master and predict the behaviour of switches. Table 1 gives the failure rate per year of the IATCT under the two defects: the switch remains open and the switch remains closed.

The definition of a priori probabilities is the most difficult step in building the model; it is based on the knowledge held by experts or the feedback. It also requires special attention because the results depend on data.

Fig. 4 Represents a BN which gives a posteriori probabilities of the variable (control of section) depending on the switch reliability.

A posteriori probabilities given in Table 3 are calculated from the a priori probabilities and CPT given in Table 2.

\subsection{DBN modelling}

DBN encodes the joint probability distribution of a time-evolving set of variables $\mathrm{V}(\mathrm{t})=\{\mathrm{V} 1(\mathrm{t}), \ldots, \mathrm{Vi}$ $(t)\}$. If we consider $t$ time slices (time step) of



Fig. 2 Control of section modelling with DBN. 


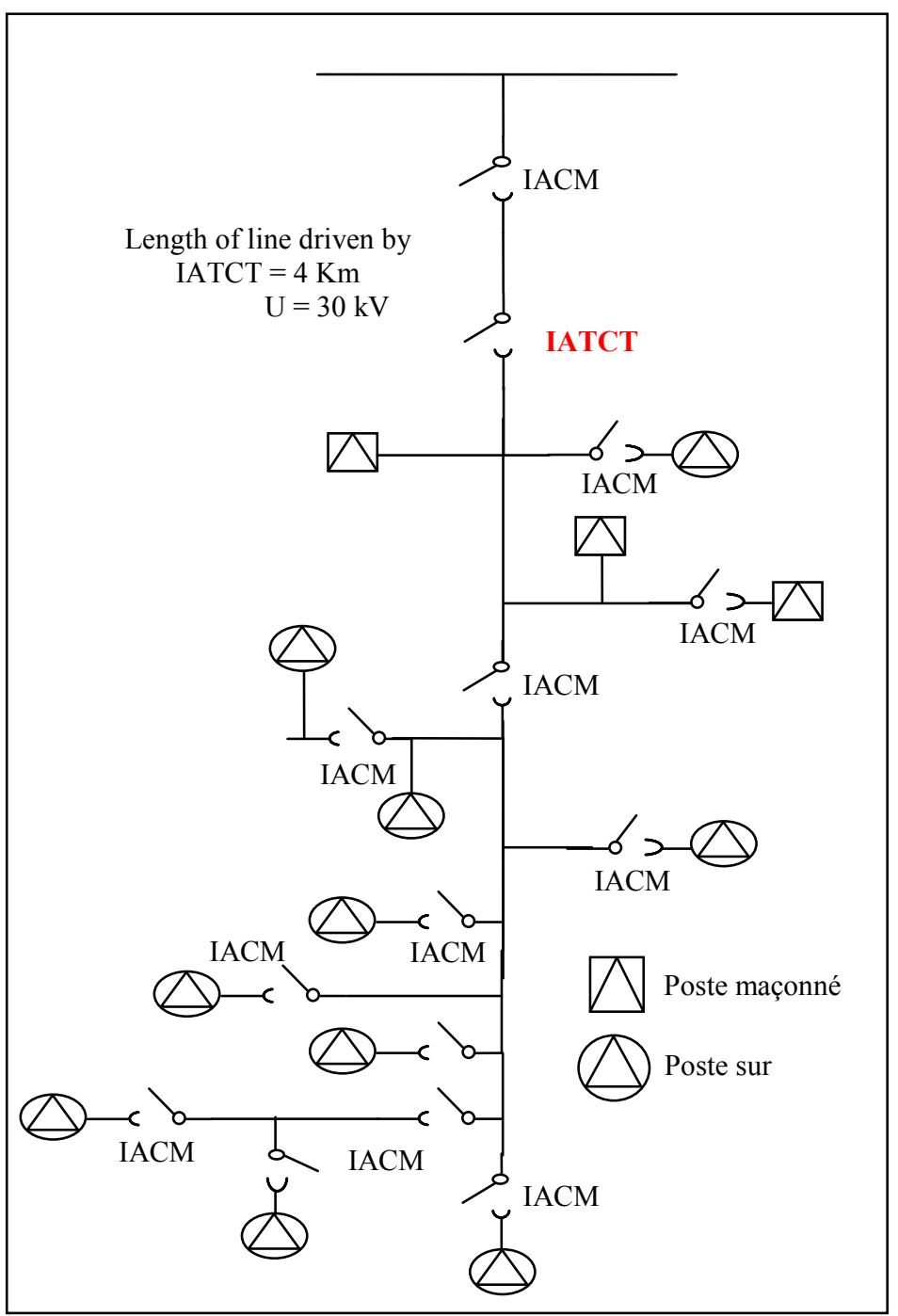

Fig. 3 Network design

Table 1 A Priori Probabilities

\begin{tabular}{ll}
\hline Failure & Failure rate ( Probabilities) \\
\hline Remains open & $\lambda=0.072$ \\
Remains closed & $\lambda=0.064$ \\
working correctly & $\lambda=0.864$ \\
\hline
\end{tabular}

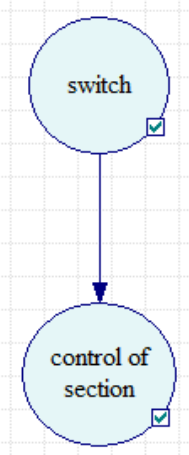

Fig. 4 Static Bayesian network modelling
Table 2 Conditional Probabilities Table

\begin{tabular}{|c|c|c|c|}
\hline \multirow[b]{2}{*}{ Control of section } & \multicolumn{3}{|l|}{ Switch } \\
\hline & $\begin{array}{l}\text { Remains } \\
\text { closed }\end{array}$ & $\begin{array}{l}\text { Remains } \\
\text { open }\end{array}$ & $\begin{array}{l}\text { Working } \\
\text { correctly }\end{array}$ \\
\hline Supplied section & 0 & 1 & 1 \\
\hline Isolated section & 1 & 0 & 0 \\
\hline
\end{tabular}

Table 3 A Posteriori Probabilities of Variable Control of Section

\begin{tabular}{ll}
\hline Control of section & A posteriori Probabilities \\
\hline Supplied section & 0.928 \\
Isolated section & 0.072 \\
\hline
\end{tabular}

variables, the DBN can be considered as a "static" BN with $\mathrm{T} \times \mathrm{i}$ variables.

The temporal probability distributions of parent variable (switch) with three state (remains open, 


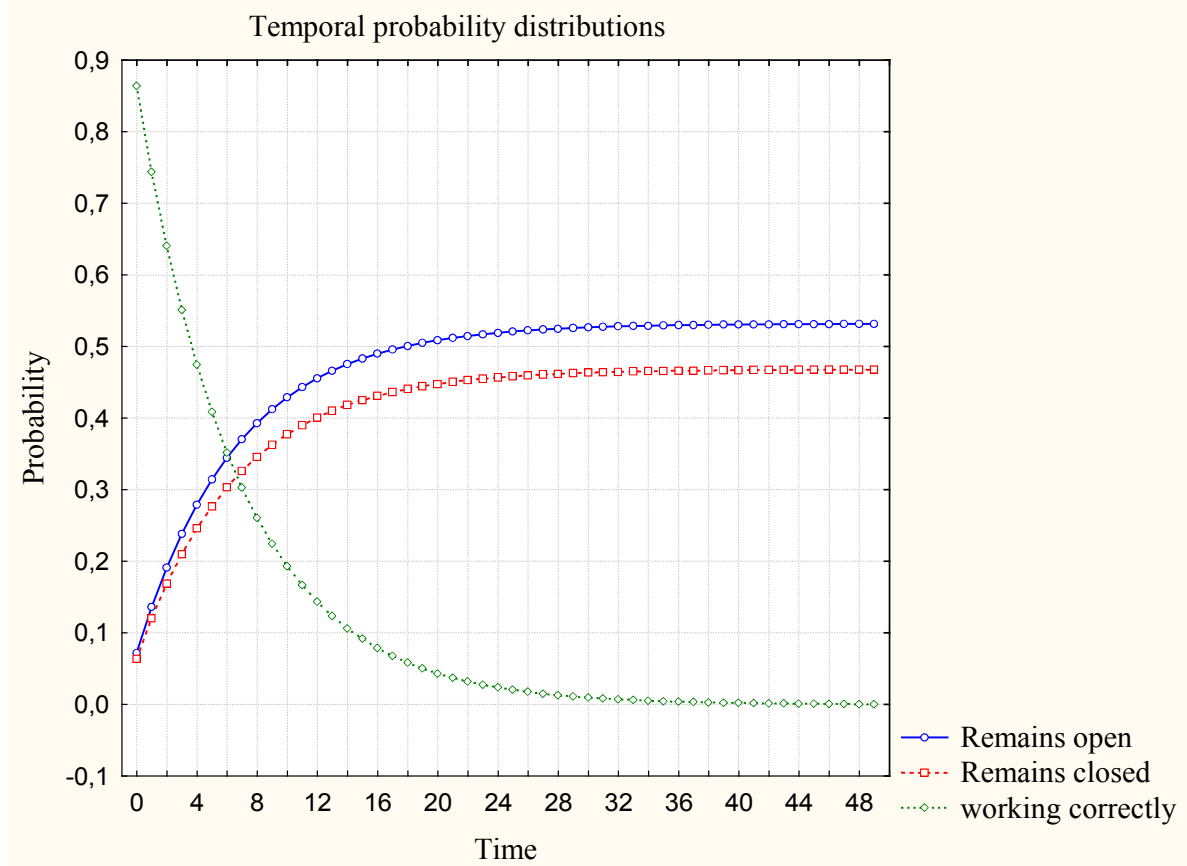

Fig. 5 Temporal probability distributions.

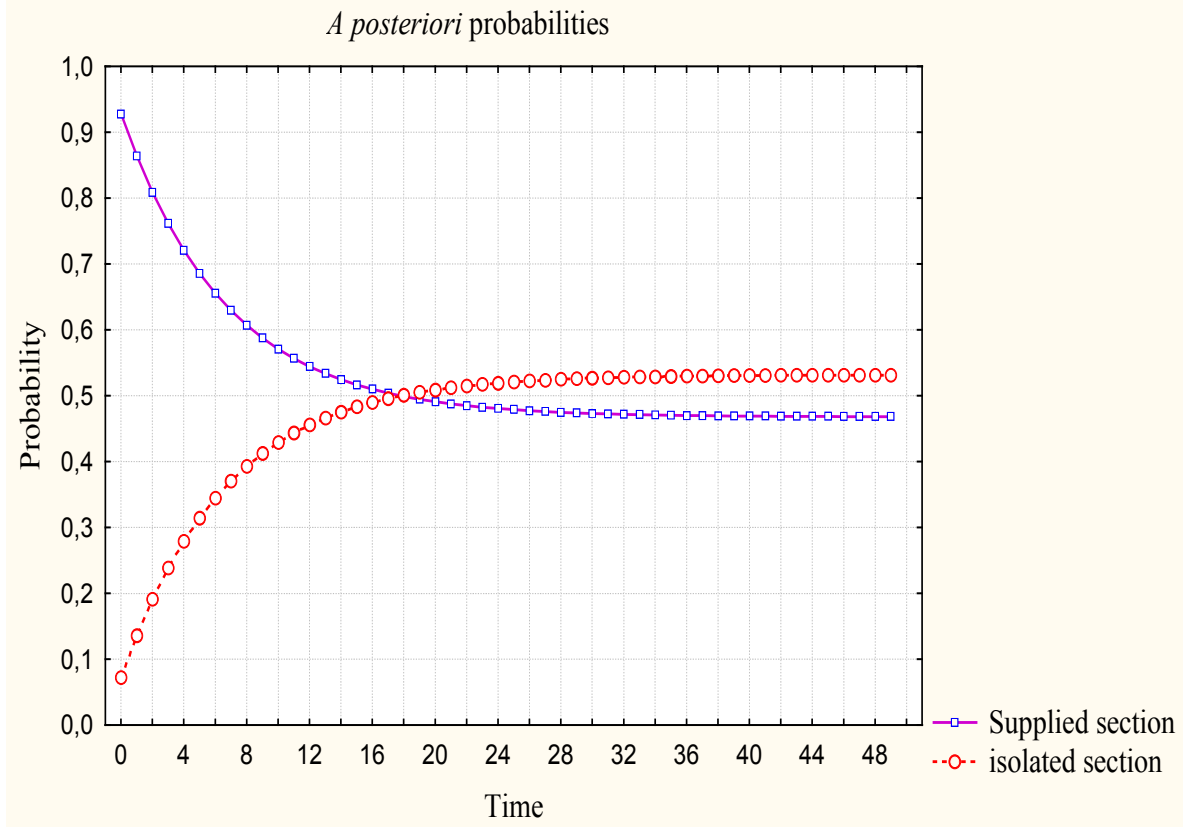

Fig. 6 A posteriori temporal probability distributions of variable control of section.

remains closed, and working correctly) are shown in fig. 5 .

Modelling the dynamic behaviour of the switch and therefore the two states (isolated section) and (Supplied section) versus time consists of 50 time steps, with each time slice contains its own variables.
The state of the section is quantified by a posteriori probability per (2); the results are presented in Fig. 6 .

This section presents the prediction results of an experimentation conducted on an IATCT feeding locality. From the results given by our model, we can extract knowledge and transform them automatically 
for probabilistic, quantitative and qualitative prediction results, these prediction results of our system allows us to anticipate different situations caused by failure in power supply system, it is very encouraging.

Indeed, our study concerns the prediction of the network state. This prediction is dynamic; it evolves throughout the operation of the network by new measures and new situation. With each year of the network operations, we could envisage his state at the future by a probability, which will be used, in the prediction of the year +1 , with these measured observations.

\section{Conclusion}

In this paper, we described an application of decision support system to the electrical distribution network operations. This system aims at helping the operators to estimate the availability rate. The decision given by this model is dynamic because it is based on the network state described in switch temporal behaviour of which the unit of time is the year. The dynamic decision system evolves and proceeds in several stages corresponding to the increasing levels of the equipments situation comprehension (in our example switch state). On each level, a set of knowledge can be generated.

We have implemented the DBNs based on fixed (at $\mathrm{t}=0$ that gives a static $\mathrm{BN}$ ) and temporal data (years of operations). The application of the developed models for behaviour prediction of cut-off devices gives good results.

The control of power distribution network includes mastery of behaviour of all static equipment such as transformer and dynamic such as the cut-off devices. Network design, equipment condition, and the human factor are elements which help to ensure quality and continuity of service in the profession of electricity distribution. In our future work, we will take over all these elements by static / dynamic models on the one hand and secondly by hybrid BNs for example to model the influence of consumption during peak hours on electrical network availability.

\section{Acknowledgment}

We would like to express our appreciations for the valuable time of anonymous reviewers spent on the review process.

\section{References}

[1] A. Bobbio, L. Portinale, M. Minichino, and E. Ciancamerla, "Improving the analysis of dependable systems by mapping fault trees into Bayesian networks," Reliability Engineering and System Safety, vol. 71, no. 3, pp. 249-260, 2001.

[2] A. Moradhhani, M.R. Haghifam, and M. Mohammadzadeh, "Bayesian estimation of overhead lines failure rate in electrical distribution systems," Electrical Power and Energy Systems, vol. 56, pp. 220-227, 2014.

[3] T. Iešmantas, and R. Alzbutas, "Bayesian assessment of electrical power transmission grid outage risk," Electrical Power and Energy Systems, vol. 58, pp. 85-90, 2014.

[4] J. Augutis, I. Zutautaite, V. Radziukynas, R. Krikstolaitis, and S. Kadisa, "Application of Bayesian method for electrical power system transient stability assessment," Electrical Power and Energy Systems, vol. 42, pp. 465-472, 2012.

[5] V. Shrivastava, and R.B. Misra, "Development of Bayesian belief network model for electrical load demand," Inetrnational Journal of System Assurance Engineering and Management, vol. 1, no. 2, pp. 170-177, 2010.

[6] A. Doulet, "Réseaux de distribution - Exploitation-," Techniques de l'ingénieur, D4 230, France, 2001.

[7] F.V. Jensen, An Introduction to Bayesian Networks. UCL Press, London, 1996.

[8] A. Lakehal, and Z. Ghemari, "Une nouvelle méthode de diagnostic basée sur la conversion d'un arbre de défaillances en réseau bayésien," in Proc. Of ICIEM'14, International Conference on Industrial Engineering and Manufacturing, Batna University, Algeria, May 11-13, 2014, pp. 507-514.

[9] V. Delcroix, M.A. Maalej, and S. Piechowiak, "Bayesian networks versus other probabilistic models for the multiple diagnosis of large devices," International Journal on Artificial Intelligence Tools, vol. 16, no. 3, pp. 417-433, 2007.

[10] E. Hadjadj Aoul, and A. Lakehal, "Combined anomalies 


\section{Bayesian Networks: Case Study of Electrical Distribution Networks}

prediction using the Bayesian theory," Quality and Reliability Engineering International, vol. 28, no. 3, pp. 363-367, 2012.

[11] A. Léger, C. Duval, P. Weber, E. Levrat, and R. Farret, "Risk analysis of complex socio-technical systems by using Bayesian network modelling". In: Workshop on Advanced Control and Diagnosis, France, 2006.

[12] P. Naim, P.H. Wuillemin, P. Leray, O. Pourret, and A. Becker, Réseaux Bayésiens. 2 ed. Eyrolles, France, 2004.
[13] H. Boudali, and J.B. Dugan, "A discrete-time Bayesian network reliability modeling and analysis framework," Reliability Engineering and System Safety, vol. 87. No. 3, pp. 337-349, 2005.

[14] P. Weber, and L. Jouffe, "Reliability modelling with dynamic Bayesian networks". 5th IFAC Symposium on Fault Detection, Supervision and Safety of Technical Processes (Safeprocess'03), Washington, D.C., USA, June 9-11, 2003. 